\title{
7-Azaindoline Auxiliary: A Versatile Attachment Facilitating Enantioselective C-C Bond-Forming Catalysis
}

\author{
Naoya Kumagai* (D) \\ Masakatsu Shibasaki* (D) \\ Institute of Microbial chemistry (BIKAKEN), 3-14-23 Kamiosaki, \\ Shinagawa-ku, Tokyo 141-0021, Japan \\ nkumagai@bikaken.or.jp \\ mshibasa@bikaken.or.jp \\ Published as part of the 50 Years SYNTHESIS - Golden Anniversary Issue
}

Received: 14.11 .2018

Accepted: 16.11.2018

Published online: 30.11 .2018

DOI: 10.1055/s-0037-1610412; Art ID: ss-2018-z0767-sr

License terms: (c)

Abstract This short review provides an overview of 7-azaindoline auxiliaries in asymmetric catalysis. 7-Azaindoline serves as a useful attachment to carboxylic acids, and the thus-formed 7-azaindoline amides are amenable to atom-economical $\mathrm{C}-\mathrm{C}$ bond-forming reactions with high stereoselectivity. The attachment is used for the sake of gaining traction in promoting the reaction of interest and can be easily removed after enantioselective reactions. Both nucleophilic and electrophilic catalyses are realized with broad tolerance for functional groups, showcasing the usefulness of 7-azaindoline auxiliaries for practical and streamlined synthesis of a wide range of acyclic chiral building blocks. Introduction

7-Azaindoline as a Key Auxiliary

7-Azaindoline Amide as a Pronucleophile

$\alpha$-Carbon-Substituted 7-Azaindoline Amide

$\alpha$-Nitrogen-Substituted 7-Azaindoline Amide

$\alpha$-Oxygen-Substituted 7-Azaindoline Amide

$\alpha$-Fluorocarbon-Substituted 7-Azaindoline Amide

$\alpha$-Halogen-Substituted 7-Azaindoline Amide

$\alpha$-Sulfur-Substituted 7-Azaindoline Amide

7-Azaindoline Amide as an Electrophile

Conjugate Addition of Butenolides

1,3-Dipolar Cycloaddition of Nitrones

Transformation of 7-Azaindoline Amide

Conclusion

Key words asymmetric catalysis, $\mathrm{C}-\mathrm{C}$ bond formation, aldol reaction, Mannich reaction, 7-azaindoline, cycloaddition

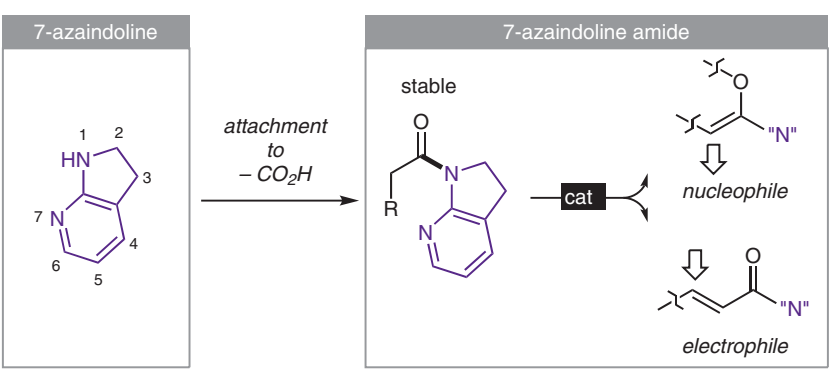

\section{Introduction}

Given the increased demand for optically active synthons in various research fields, the focus of asymmetric catalysis has shifted from lab-scale demonstration to sophistication with a practical robustness. To access specific enantioenriched synthons of interest with rapid synthesis and overall efficiency, $\mathrm{C}-\mathrm{C}$ bond-forming reactions furnishing $\mathrm{sp}^{3}$-carbon connectivity with decent control of the stereoselectivity are ideal. Our research group has focused on devising a versatile synthetic tool to access a range of broadly applicable chiral building blocks, in which the designed catalyst is solely responsible for promoting the reactions without the use of reagents. Our continued research in this context over the last 5 years has shown that 7 -azaindoline is a versatile substrate attachment that allows for a variety of enantioselective reactions. This short review summarizes the utility of 7-azaindoline units in asymmetric catalysis.

\section{7-Azaindoline as a Key Auxiliary}

In addition to the obviously important factors, e.g. reactivity and stereoselectivity, chemoselectivity is indispensable for enabling the reaction of interest in the ensemble of chemical species bearing different functional groups. Although chemoselectivity that is solely exerted by a catalyst is ideal, it may diminish control of the chemoselectivity, leading to undesired side reactions. We searched for a substrate 'attachment' that: 1 . would enable stabilization of the substrate itself to suppress uncontrolled reactions (side-reactions, low stereoselectivity); 2 . would switch on a hidden 


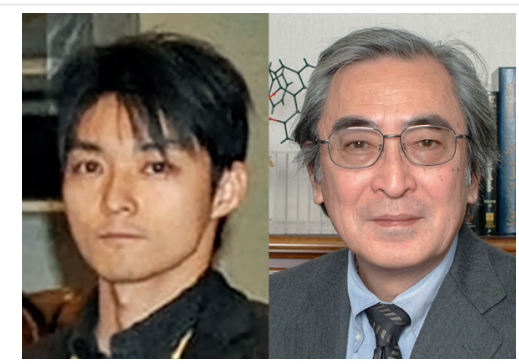

Naoya Kumagai was born in 1978 and raised in Ibaraki, Japan. After receiving his Ph.D. in Pharmaceutical Sciences at the University of Tokyo in 2005 under the supervision of Prof. Masakatsu Shibasaki, he pursued postdoctoral studies in the laboratory of Prof. Stuart L. Schreiber at Harvard University in 2005-2006. He moved to Prof. Shibasaki's group at the University of Tokyo as an assistant professor in 2006. He is currently a chief researcher at the Institute of Microbial Chemistry, Tokyo. He is a recipient of the Pharmaceutical Society of Japan Award for Young Scientists (2010); Banyu Chemist Award (2012); Young Scientists' Prize; a commendation for Science and Technology by the Minister of Education, Culture, Sports, Science and Technology (2013); Mistui Chemicals Catalysis Science Award of Encouragement (2014); and the Merck-Banyu Lectureship Award (MBLA) in 2017. His research interests include the development of new methods for catalysis and their application to bioinspired dynamic processes.

Masakatsu Shibasaki received his Ph.D. from the University of Tokyo in 1974 under the direction of the late Professor Shun-ichi Yamada before doing postdoctoral studies with Professor E. J. Corey at Harvard University. In 1977, he returned to Japan and joined Teikyo University as an associate professor. In 1983, he moved to Sagami Chemical Research Center as a group leader, and in 1986 he assumed a professorship at Hokkaido University before returning to the University of Tokyo as professor in 1991. He is currently the Director of the Institute of Microbial Chemistry, Tokyo. He has received several prestigious awards, including the Fluka Prize (Reagent of the Year, 1996); the Elsevier Award for Inventiveness in Organic Chemistry (Tetrahedron Chair) (1998); the Pharmaceutical Society of Japan Award (1999); the ACS Award (Arthur C. Cope Senior Scholar Award) (2002); the National Prize of Purple Ribbon (2003); the Japan Academy Prize (2005); the ACS Award for Creative Work in Synthetic Organic Chemistry (2008); the Centenary Medal and Lectureship (2008); the Prelog Award Medal (2008); the Special Award, the Society of Synthetic Organic Chemistry, Japan (2010); the Noyori Prize (2012), and others. His research interests include asymmetric catalysis and medicinal chemistry of biologically significant compounds.

reactivity of stable (unreactive) substrates by a specific trigger (catalyst) to elicit high-fidelity chemoselectivity; and 3. would endow the product with sufficient stability to warrant reliable isolation and handling (Scheme 1). We found that these requirements were fulfilled by 7 -azaindoline amides $\mathbf{2}$, in which the amide functionality adds thermodynamic stability and crystallinity while the pyridyl nitrogen can be activated by a certain trigger in a catalytic manner. Intriguingly, the 7-azaindoline $\mathbf{1}$ attachment renders the corresponding amide 2 reactive with a specific catalytic system in both nucleophilic (as enolate) and electrophilic (as conjugate addition acceptor) reaction manifolds, as described in the following sections.

These structurally related amides are intractable under catalytic conditions specifically designed for 7-azaindoline amide $\mathbf{2}$, showcasing its chemoselective activation.

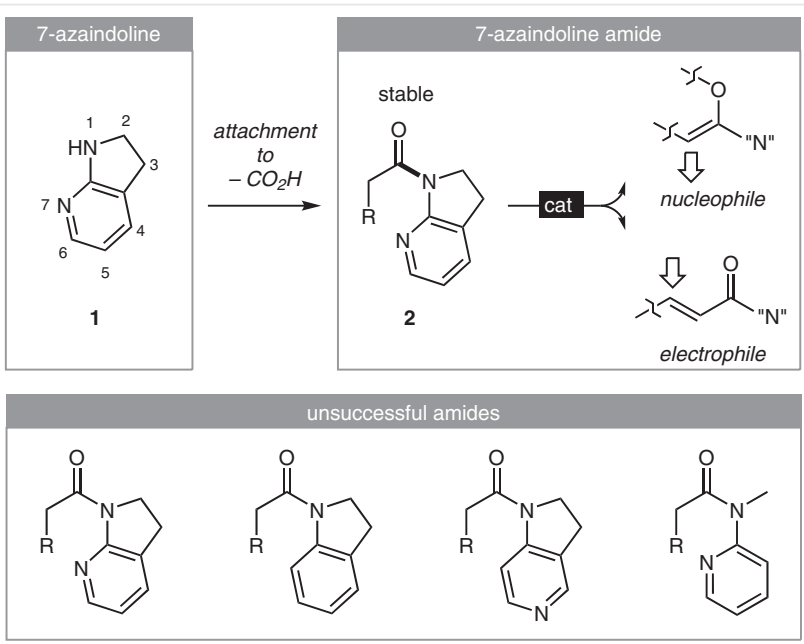

Scheme 1 7-Azaindoline and 7-azaindoline amide; structurally related amides show no reactivity under the same catalytic conditions

\section{7-Azaindoline Amide as a Pronucleophile}

Based on X-ray crystallography and ${ }^{1} \mathrm{H}$ NMR, the 7azaindoline amides $\mathbf{2}$ assume an $E$-conformation as both a solid and in solution phase, respectively (Scheme 2a). The $E$-amide conformation is readily inverted to the activated form of $Z$-amide upon the addition of a soft Lewis acidic metal complex. The chelated structure revealed by X-ray crystallography facilitates enolization upon the combined use of a mild Brønsted base as a co-catalyst, efficiently driving direct-type aldol and Mannich-type reactions on the chiral ligand of metal cations in an asymmetric environment. ${ }^{1,2} \mathrm{~A}$ notable feature of 7 -azaindoline amides 2 as enolate precursors is the wide variety of applicable $\alpha$-substituents; basically, all the elements [ $\alpha$-alkyl (C), ${ }^{3} \alpha$-fluoroalkyl $\left(\mathrm{R}^{\mathrm{F}}\right){ }^{4} \alpha$-azido $(\mathrm{N}),{ }^{5} \alpha$-benzyloxy (O), ${ }^{6} \alpha$-halo $(\mathrm{F}, \mathrm{Cl}, \mathrm{Br}, \mathrm{I}){ }^{7}$ and $\alpha$-methylsulfanyl $\left.(S)^{8}\right]$ used in standard organic synthesis can be installed, highlighting its particular utility for providing tailor-made chiral building blocks with the pendant $\alpha$-substituent of interest. The cooperative catalytic system $^{9}$ comprising a soft Lewis acid and Brønsted base has three variants depending on the Brønsted base: Type A: $\left[\mathrm{Cu}\left(\mathrm{CH}_{3} \mathrm{CN}\right)_{4}\right] \mathrm{PF}_{6} /$ chiral P-ligand/Barton's base; Type B: mesitylcoppe ${ }^{10} /$ chiral P-ligand; and Type C: mesitylcopper/chiral P-ligand/phenol derivatives (Scheme 2b). Type A is an archetypal catalyst cocktail with broad competency in independent functions of the $\mathrm{Cu}(\mathrm{I}) / \mathrm{P}$-ligand complex and Barton's base, allowing for recognition/activation of the 7azaindoline amide functionality and deprotonation of the activated amide, respectively. The Type B catalyst utilizes oligomeric mesitylcopper as a Brønsted base at the initial trigger of the catalysis to form a ligated $\mathrm{Cu}(\mathrm{I})$-enolate, which undergoes $\mathrm{C}-\mathrm{C}$ bond formation with $\mathrm{C}=\mathrm{O}$ (aldehyde) 
or $\mathrm{C}=\mathrm{N}$ (imine) type electrophiles. The thus-formed $\mathrm{Cu}(\mathrm{I})-$ aldolates or $\mathrm{Cu}(\mathrm{I})$-amides act as a soft Lewis acid/Brønsted base cooperative catalyst to drive the following catalytic cycle. The addition of phenol derivatives to the Type B catalyst mixture gives the Type $C$ catalyst, which is characterized by a ligated $\mathrm{Cu}(\mathrm{I})$-aryloxide complex.

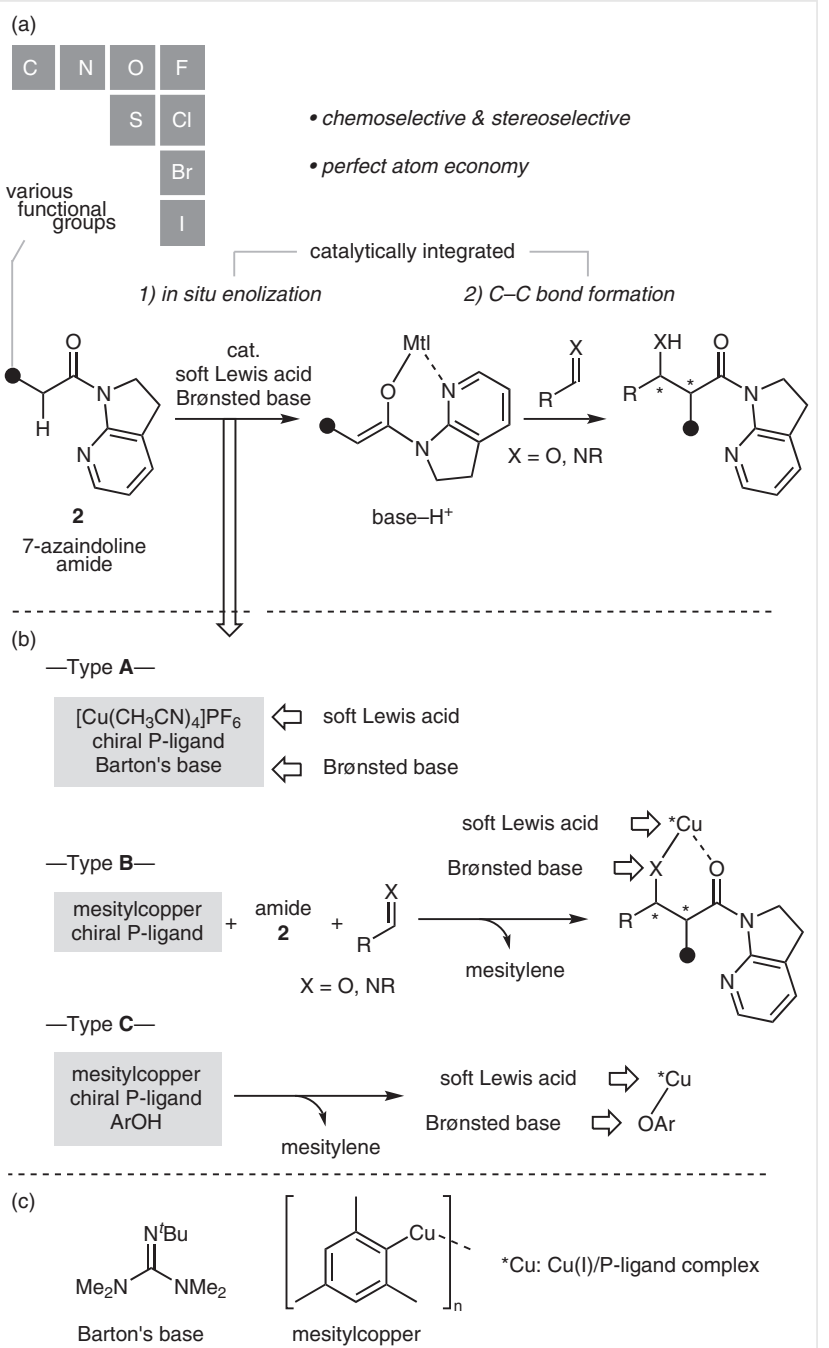

Scheme 2 (a) 7-Azaindoline amide as an enolate precursor. (b) Variants of the cooperative catalyst used for nucleophilic activation of 7azaindoline amide; (c) Structures of reagents/ligands.

\section{1 $\alpha$-Carbon-Substituted 7-Azaindoline Amide}

The C3 propionate unit is ubiquitously found in a number of biologically active natural products and therapeutic agents. The aldol- and Mannich-type reactions offer a sim- ple, yet powerful method for constructing carbon frameworks containing the propionate unit. The facilitated enolization capability of 7-azaindoline propionamide $\mathbf{2 a}$ emerged as a proficient pronucleophile in this regard, allowing for enantioselective coupling with aldehydes $\mathbf{4}$ and imines 5. ${ }^{3}$ The combined use of Lewis acid [chiral $\mathrm{Cu}(\mathrm{I})$ complexes] and Brønsted base (Barton's base or phenoxides) at temperatures ranging from $-40{ }^{\circ} \mathrm{C}$ to room temperature promoted the catalytic enolization of $\mathbf{2 a}$ and subsequent addition to electrophiles. Systematic studies revealed that the privileged ligand depended on subtle changes of the electrophiles, which likely reflects the fact that the open-transition state is operative in this catalysis. The sterics of the amide moiety prefer the formation of a $Z$ configured $\mathrm{Cu}(\mathrm{I})$-enolate, where $\mathrm{Cu}(\mathrm{I})$ is coordinatively saturated and the face-selection of the electrophiles is strongly affected by the chiral ligand used. Although $\alpha$-sp ${ }^{3}$ aldehydes and enals were found to be intractable, ynals $\mathbf{4}_{\text {ynal }}$ and aromatic aldehydes $\mathbf{4}_{\mathrm{Ar}}$ exhibited high reactivity to afford the desired aldol products with high diastereo- and enantioselectivity (Schemes 3 and 4). ${ }^{3 \mathrm{~b}}$ Due to the likely involvement of the open-transition state, diverted diastereoselectivity is observed by the combination of the chiral ligand and aldehydes. $(R)$-Trimethoxy-Biphep (L1) was identified as a preferred ligand in the Type $C$ catalyst to steer the high antiand enantioselectivity to deliver propargylic alcohol 6 containing the synthetically versatile propionate unit (Scheme 3). Intriguingly, with the Type B catalyst using $(S, S)-\mathrm{Ph}-\mathrm{BPE}$ (L2) as a chiral ligand, aromatic aldehydes $\mathbf{4}_{\mathrm{Ar}}$ react with 7azaindoline propionamide $\mathbf{2 a}$ to give syn-configured aldol products 7 .

The applicability of 7-azaindoline propionamide $\mathbf{2 a}$ for a Mannich-type reaction ${ }^{11}$ expands the accessible chiral building blocks containing the propionate unit. ${ }^{3 \mathrm{a}} \alpha-\mathrm{sp}^{2} \mathrm{~N}$ Boc imines 5 derived from aromatic aldehydes and enal exhibit good reactivity. The Type A catalyst proved optimal with $\mathbf{L 1}$ as a chiral ligand, which is also used in the aldol reaction with ynals $\mathbf{4}_{\text {ynal }}$, producing anti-configured $\beta$-amino acid derivatives anti-8 (Scheme 5). The aldol and Mannich adducts share an identical absolute configuration, suggesting that the face selection of the $\mathrm{Cu}(\mathrm{I})$-enolate complex as well as that of $\mathrm{C}=\mathrm{O}$ or $\mathrm{C}=\mathrm{N}$ is operative in a similar manner via an open-transition state. By switching the chiral ligand from $\mathbf{L 1}$ to Walphos-type ligand $\mathbf{L 3}$, syn-adducts syn-8 are obtained as major diastereomers with high enantioselectivity (Scheme 6). Of note, other than C3 propionamide, C2 acetamide and C4 butyramide are also competent in this catalytic Mannich protocol, albeit with somewhat lower reactivity and stereoselectivity. 


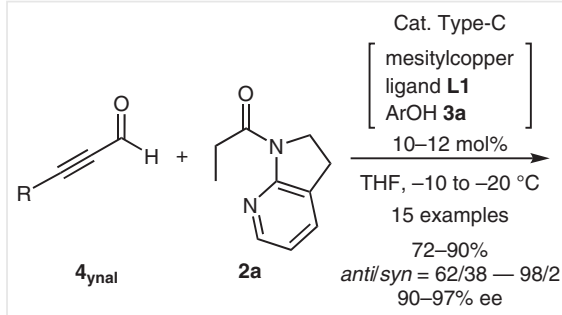<smiles>[R]C#CC(O)[C@H](C)C(=O)N1CCc2cccnc21</smiles><smiles>COc1cccc(P)c1-c1c(P)cccc1P(C)(=O)O</smiles>

L1: $(R)$-trimethoxy-Biphep $\left(\mathrm{Ar}=3,4,5-(\mathrm{MeO})_{3} \mathrm{C}_{6} \mathrm{H}_{2}\right)$

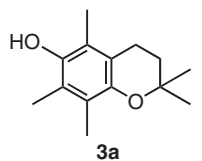

Scheme 3 Direct catalytic asymmetric anti-selective aldol reaction of 7-azaindoline propionamide with ynals

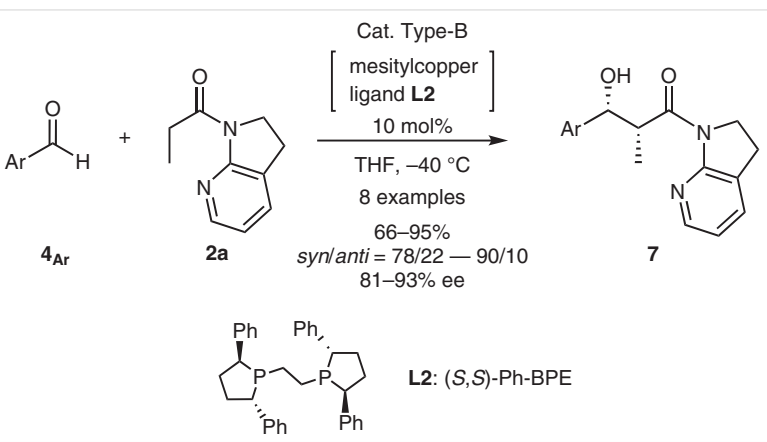

Scheme 4 Direct catalytic asymmetric syn-selective aldol reaction of 7-azaindoline propionamide with aromatic aldehydes

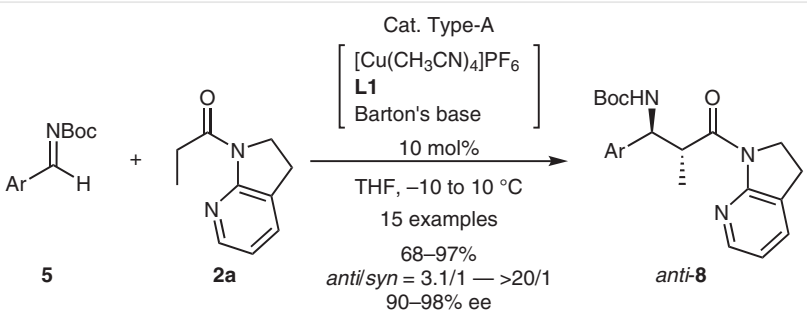

Scheme 5 Direct catalytic asymmetric anti-selective Mannich-type reaction of 7-azaindoline propionamide with $\mathrm{N}$-Boc imines using $(R)$-trimethoxy-Biphep as a ligand

\section{2 $\alpha$-Nitrogen-Substituted 7-Azaindoline Amide}

Given the particular utility of the products as natural and unnatural $\alpha$-amino acid derivatives, the $\alpha$-nitrogenfunctionalized acetic acid unit has broad synthetic value as a pronucleophile in enolate chemistry. Azido-functionalized 7-azaindoline amide $\mathbf{2 b}$, which exhibits facile enolization capability and minimized steric bias, is best suited for this purpose. ${ }^{5}$ The simple Type B catalyst system, comprising mesitylcopper and $(R, R)$-Ph-BPE (L4), efficiently promotes the aldol reaction of ortho-substituted aromatic al-

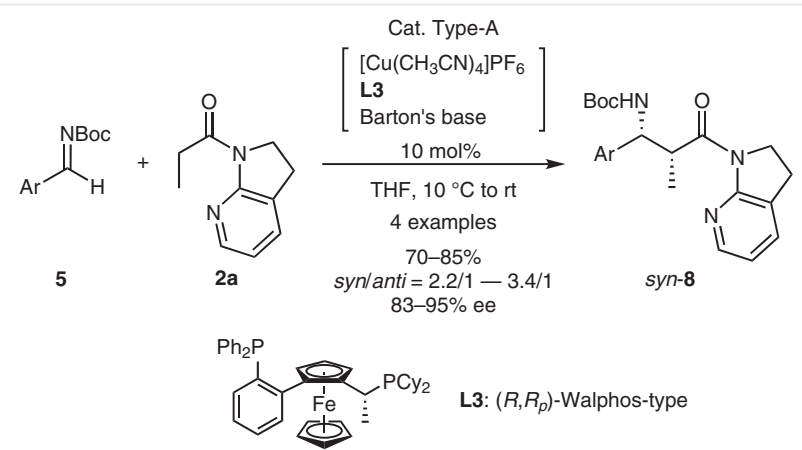

Scheme 6 Direct catalytic asymmetric syn-selective Mannich-type reaction of 7-azaindoline propionamide with $N$-Boc imines using a Walphos-type ligand

dehydes $\mathbf{4}_{\text {Ar-ortho }}$ and ynals $\mathbf{4}_{\text {ynal }}$ in an anti-selective manner with high enantioselectivity (Scheme 7$).{ }^{5 b}$ Face selection of the approaching aldehydes is highly sensitive to subtle changes in steric factors; aromatic aldehydes without ortho-substituents give a syn-configured product with an identical absolute configuration at the $\alpha$-position under identical reaction conditions. syn-Adducts of ortho-substituted aromatic aldehydes $\mathbf{4}_{\text {Ar-ortho }}$ are accessible by switching the chiral ligand from $\mathbf{L} 4$ to $(R)$-xyl-Binap $\mathbf{L 5}$ (Scheme 8 ). The diastereoselectivity is lowest with 0 -fluorobenzaldehyde, suggesting that the sterics are crucial for the face selection of aldehydes.

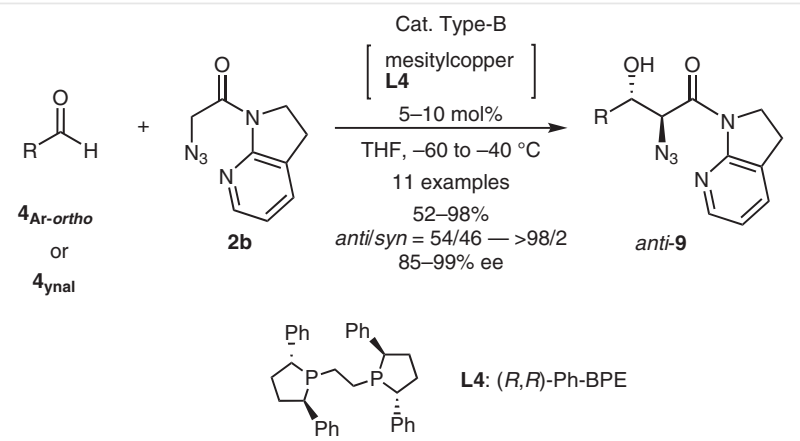

Scheme 7 Direct catalytic asymmetric anti-selective aldol reaction of $\alpha$-azido 7-azaindoline acetamide with ortho-substituted aldehydes and ynals using $(R, R)$-Ph-BPE as a ligand

$\alpha$-Trifluoromethyl ynones $\mathbf{1 0}$ are also incorporated as applicable electrophiles, affording densely functionalized propargylic tertiary alcohols 11 (Scheme 9$).{ }^{4 \mathrm{e}}$ In this particular example, catalyst Types A-C failed to afford products with synthetically useful stereoselectivity, which was addressed by utilizing instead the $\mathrm{Cu}$ (II) complex of bishydroxamic acid (BHA) ligands developed by Yamamoto and co-workers. ${ }^{12}$ Kinetic studies revealed second-order dependency on the BHA-Cu(II) complex, suggesting that the BHA-Cu(II) complex has a dual role to activate 7-azaindoline amide $\mathbf{2 b}$ as a Lewis acid and to deprotonate 7-azaindoline amide $\mathbf{2 b}$ as a Brønsted base. 


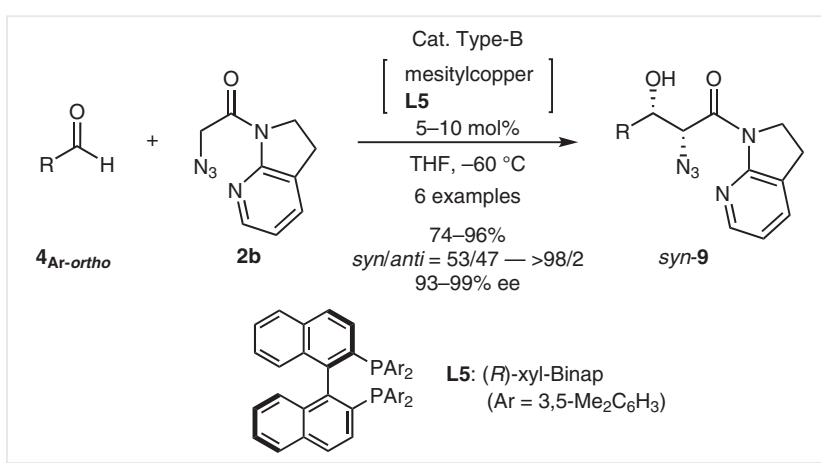

Scheme 8 Direct catalytic asymmetric syn-selective aldol reaction of $\alpha$-azido 7-azaindoline acetamide with ortho-substituted aldehydes using $(R)$-xyl-Binap as a ligand

$$
\text { (2) }
$$

Scheme 9 Direct catalytic asymmetric syn-selective aldol reaction of $\alpha$-azido 7-azaindoline acetamide with $\alpha$-trifluoromethyl ynone

Azido-functionalized 7-azaindoline amide $\mathbf{2 b}$ also contributes to the synthesis of 1,2-diamino acid units via a Mannich-type reaction with $N$-diphenylthiophosphinoyl imines 12. ${ }^{\text {a }}$ The Type A catalyst with $(R)$-xyl-Segphos $\mathbf{L 6}$ gave the desired Mannich adducts 13 with high anti- and enantioselectivity (Scheme 10). A soft Lewis basic thiophosphinoyl group on the imine nitrogen is essential for high stereoselectivity.

\section{3 $\alpha$-Oxygen-Substituted 7-Azaindoline Amide}

The use of $\alpha$-oxygen-functionalized 7-azaindoline amide $\mathbf{2 c}$ in a Mannich-type reaction provides enantioenriched 1,2-amino alcohol units 14 (Scheme 11), ${ }^{6}$ in which the positions of the nitrogen and oxygen are swapped compared with product $\mathbf{9}$. The reaction can be conveniently

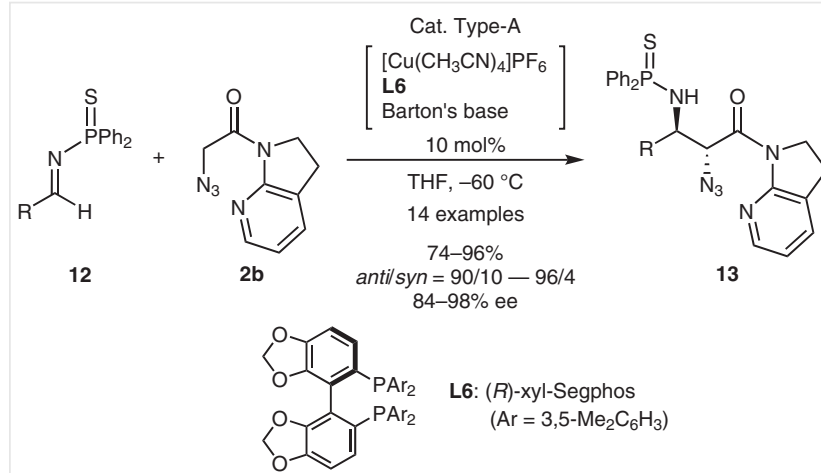

Scheme 10 Direct catalytic asymmetric anti-selective Mannich-type reaction of $\alpha$-azido 7-azaindoline acetamide with $\mathrm{N}$-diphenylthiophosphinoyl imines

conducted at room temperature using a Type A catalyst, affording syn-adducts 14 with high stereoselectivity. This reaction is particularly useful for the enantioselective synthesis of the side chain of docetaxel and paclitaxel $(\mathrm{Ar}=\mathrm{Ph})$, which are highly potent anticancer pharmaceuticals. ${ }^{13}$

\section{4 $\alpha$-Fluorocarbon-Substituted 7-Azaindoline Am- ide}

Given the established beneficial effects of introducing fluorine into active pharmaceutical ingredients, ${ }^{14}$ methods for fluorination have gained increasing attention. ${ }^{15}$ The 7 azaindoline attachment has particular utility for providing optically active fluorinated compounds with functional groups. $\alpha$-Trifluoromethyl 7-azaindoline amide $2 \mathbf{d}$ serves as a fluorinated pronucleophile that can be coupled with $N$ Boc imines 5 via direct and catalytic enolization with a Type A catalyst using $(R)$-DIPA-Biphep ligand L7 (Scheme 12). ${ }^{\text {a, b }}$ In general, $\beta$-fluorinated carbonyl compounds are elusive

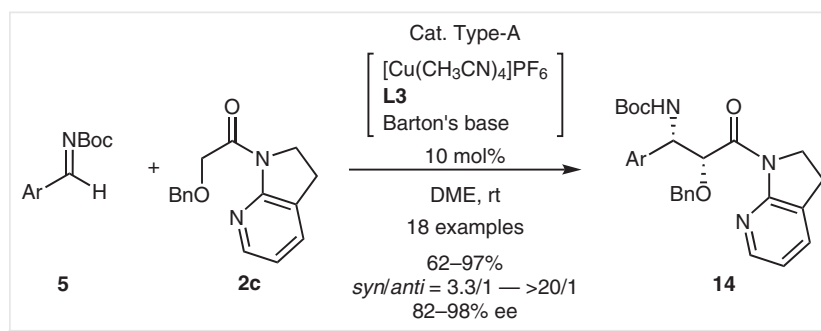

Scheme 11 Direct catalytic asymmetric syn-selective Mannich-type reaction of $\alpha$-benzyloxy 7 -azaindoline acetamide with $N$-Boc imines 
enolate precursors due to rapid defluorination, which is effectively circumvented for 7-azaindoline amide $\mathbf{2 d}$ by chelate formation with the chiral $\mathrm{Cu}(\mathrm{I})$ complex through the pyridyl nitrogen.

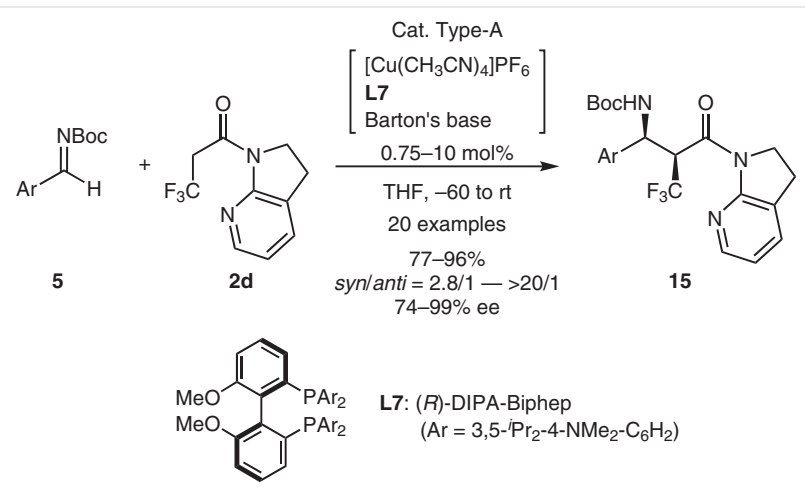

Scheme 12 Direct catalytic asymmetric syn-selective Mannich-type reaction of $\alpha$-trifluoromethyl 7 -azaindoline acetamide with $\mathrm{N}$-Boc imines

The Mannich reaction of $\alpha$-trifluoromethyl 7-azaindoline amide with $N$-Boc imines 5 using ligand $\mathbf{L 7}$ affords $\beta$ amino acid derivative $\mathbf{1 5}$ bearing an $\alpha$-trifluoromethyl group at the stereogenic carbon. The identical catalytic system is competent to engage more fluorinated 7-azaindoline amide $\mathbf{2} \mathbf{e}$ in the Mannich-type reaction. The thus-produced enantioenriched adducts $\mathbf{1 6}$ contain the fully fluorinated propionate unit, a compound not so easily accessed by other methods (Scheme 13). It is worth noting that $\alpha$-pentafluoroethyl and $\alpha$-(bromo)tetrafluoroethyl groups are also tolerated in this enantioselective Mannich reaction protocol by switching chiral ligand from $\mathbf{L 7}$ to $\mathbf{L 6}$.

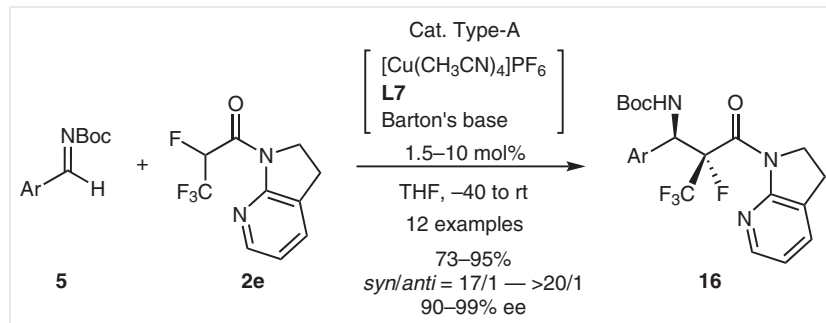

Scheme 13 Direct catalytic asymmetric syn-selective Mannich-type reaction of $\alpha$-fluoro- $\alpha$-trifluoromethyl 7-azaindoline acetamide with $\mathrm{N}$ Boc imines

Although aldehydes are intractable electrophiles in the reaction with $\alpha$-trifluoromethyl 7-azaindoline amide $\mathbf{2 d}$, the aldol reaction with arylglyoxal hydrates $\mathbf{1 7}$ proceeds smoothly to give dicarbonyl products 18 (Scheme 14). ${ }^{4 \mathrm{~d}}$ Type A catalyst with DBU and $\left(R, R_{p}\right)$-Taniaphos $\mathbf{L 8}$ is optimal for providing anti-configured aldol adducts 18. A mixed solvent system is applied to attain a homogeneous reaction mixture with sparingly soluble glyoxal hydrates 17.

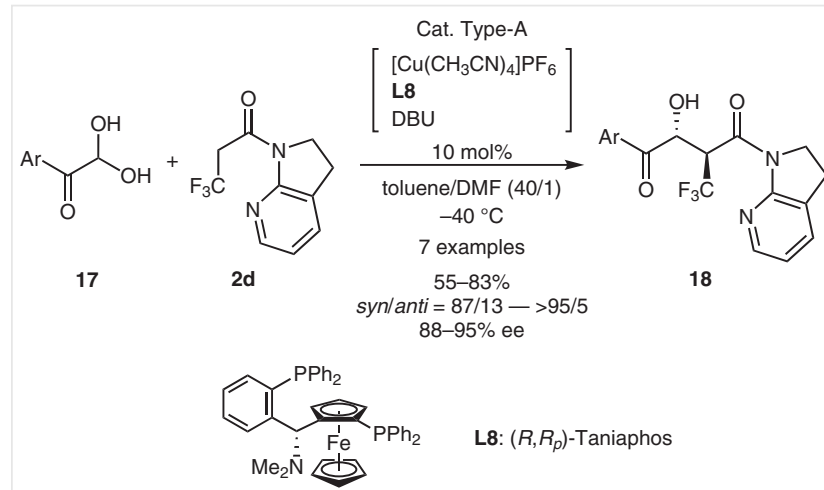

Scheme 14 Direct catalytic asymmetric anti-selective aldol reaction of $\alpha$-trifluoromethyl 7-azaindoline acetamide with glyoxals

\section{5 $\alpha$-Halogen-Substituted 7-Azaindoline Amide}

The features of the 7-azaindoline enolate surrogate are best represented by $\alpha$-halo 7 -azaindoline amide $\mathbf{2 f}$. The $\mathrm{C}-$ halogen bond next to the carbonyl functionality is generally labile and frequently undergoes uncontrolled dehalogenation.

Due to the stable nature of 7-azaindoline amides, the Chalogen bond of $\mathbf{2 f}$ and Mannich adducts $\mathbf{1 9}$ is intrinsically stable and undesired dehalogenation is not observed, even under the reaction conditions (Schemes 15 and 16). ${ }^{7} \mathrm{~N}$-Bocimines $\mathbf{5}$ are the most suitable electrophiles in this Mannich-type reaction, which is efficiently promoted by the Type A catalyst with Walphos-type ligand L3. Similarly, face selection of aromatic imines is diverted by the presence of ortho-substituents; ortho-substituted imines prefer synproducts syn-19 and others give anti-configured products anti-19 with high enantioselectivity (Scheme 15). Aliphatic imines are successfully incorporated with a different ligand L1, albeit with slightly lower diastereoselectivity (Scheme 16).

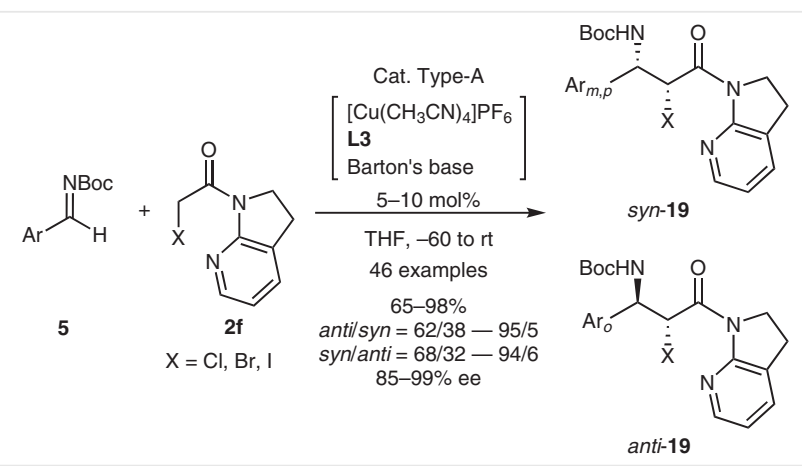

Scheme 15 Direct catalytic asymmetric anti- and syn-selective Mannich-type reaction of $\alpha$-halogenated 7 -azaindoline acetamide with aromatic $\mathrm{N}$-Boc imines 


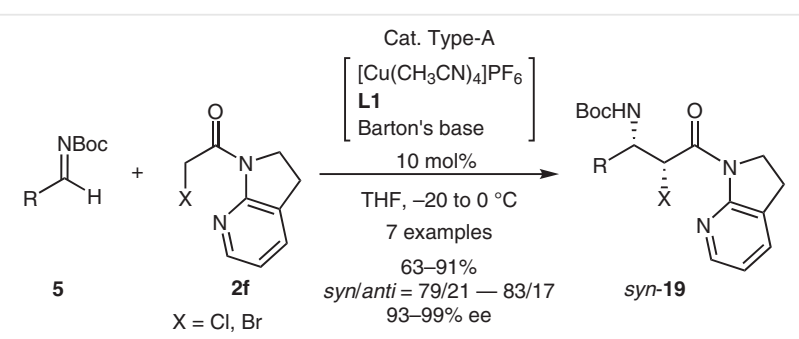

Scheme 16 Direct catalytic asymmetric syn-selective Mannich-type reaction of $\alpha$-halogenated 7-azaindoline acetamide with aliphatic $N$-Boc imines

\section{6 $\alpha$-Sulfur-Substituted 7-Azaindoline Amide}

The remaining indispensable element commonly found in biologically active natural products and therapeutics is sulfur. $\alpha$-Methylsulfanyl 7 -azaindoline acetamide $\mathbf{2 g}$ is accommodated in direct enolization chemistry and produces sulfur-containing, optically active chiral building blocks (Scheme 17). ${ }^{8}$ Likely due to the soft Lewis basic character of the sulfur atom, a distinct catalytic system employing $\mathrm{Ag}(\mathrm{I})$ cation as a Lewis acid was identified in this specific reaction. While $\mathrm{Ag}(\mathrm{I}) /(R, R)-\mathrm{Ph}-\mathrm{BPE} \mathbf{L} \mathbf{4}$ gives an insoluble complex in THF, the addition of LiOTf leads to a homogeneous mixture containing a dimeric complex, $\mathrm{Ag}_{2}(\mathbf{L} 4)_{2}(\mathrm{OTf})_{2}$, as the dominant species, as characterized by NMR and X-ray crystallographic analysis. The dimeric complex is in equilibrium with monomeric species, which is facilitated by the addition of the soft Lewis basic substrate $\alpha$-methylsulfanyl 7-azaindoline acetamide $\mathbf{2 g}$, as evidenced by ESI-MS. Interaction of the monomeric $\mathrm{Ag}(\mathrm{I})$ complex and $\mathbf{2 g}$ enhances the acidity of $\mathbf{2 g}$, en route to catalytic deprotonation by $\mathrm{Li}\left(\mathrm{OC}_{6} \mathrm{H}_{4}-p\right.$-OMe $)$. The aldol reaction with aliphatic aldehydes $\mathbf{4}$ proceeds in a syn-selective fashion with high enantioselectivity, showcasing its highly chemoselective nature to in situ generate the amide enolate exclusively. Of note, this aldol protocol tolerates the reaction using aromatic aldehydes $\mathbf{4}_{\mathrm{Ar}}$ and inverted diastereoselectivity (anti) is observed (Scheme 18). The reaction proceeds much faster than with aliphatic aldehydes, and simple application of the optimal conditions results in significantly lower enantioselectivity due to an extensive retro-aldol reaction. 2-(Methylthio)ethanol (21) was identified as an effective additive that acts as a dummy product to suppress the re-entry of the product anti-20 into the catalytic cycle. With a 10 -fold excess of $\mathbf{2 1}$ in relation to the catalyst, anti-aldol adducts anti-20 are obtained reliably with high stereoselectivity.

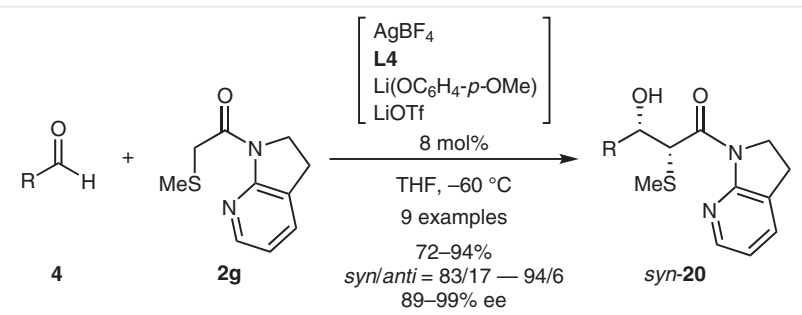

Scheme 17 Direct catalytic asymmetric syn-selective aldol reaction of $\alpha$-methylsulfanyl 7-azaindoline acetamide with aliphatic aldehydes

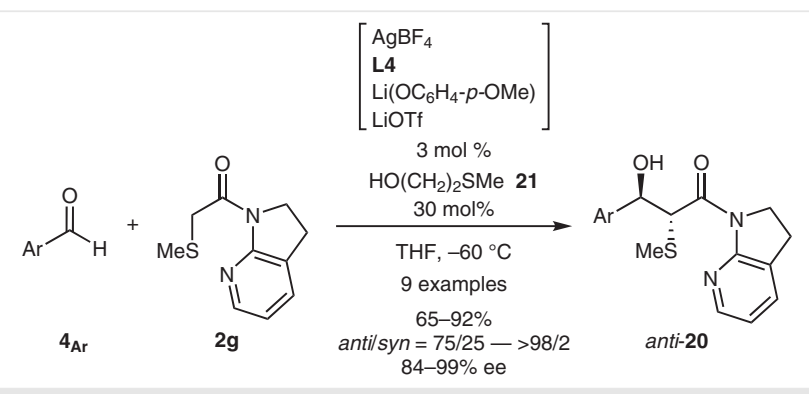

Scheme 18 Direct catalytic asymmetric anti-selective aldol reaction of $\alpha$-methylsulfanyl 7-azaindoline acetamide with aromatic aldehydes

\section{7-Azaindoline Amide as an Electrophile}

\subsection{Conjugate Addition of Butenolides}

Besides the facilitated enolization capability driven by chelate activation through the azaindoline attachment summarized in Section 3, the identical activation mode is effective for electrophilic activation of $\alpha, \beta$-unsaturated 7azaindoline amide $\mathbf{2 2}$ (Scheme 19 ). ${ }^{16}$ In a similar manner, 22 prefers the $E$-amide conformation, as confirmed by Xray crystallographic analysis. This conformation is also favored in solution phase, as evidenced by the unusually downfielded $\alpha$-proton $\left(\mathrm{H}_{\alpha}\right)$ due to hydrogen-bonding interactions with the pyridyl nitrogen. The switch to the $Z$-amide conformation is realized by the addition of the $\mathrm{Cu}(\mathrm{I})$ complex, which is now used to enhance the electrophilicity of the $\beta$-carbon in an unsaturated framework. Barton's base in the Type A catalyst allows for deprotonative activation of pronucleophiles, and butanolides $\mathbf{2 3}$ bearing a variety of substituents are widely applicable for catalytic coupling with $\alpha, \beta$-unsaturated 7-azaindoline amide 22 . While $(R)$ xyl-Binap L5 is optimal for bulkier butenolides $23\left(R^{2} \neq H\right)$, less sterically demanding $\mathbf{2 3}(\mathrm{R}=\mathrm{H})$ requires bulkier ligand $(R)$-DTBM-Segphos $\mathbf{L} 9$ to realize efficient traction on stereoselectivity. 


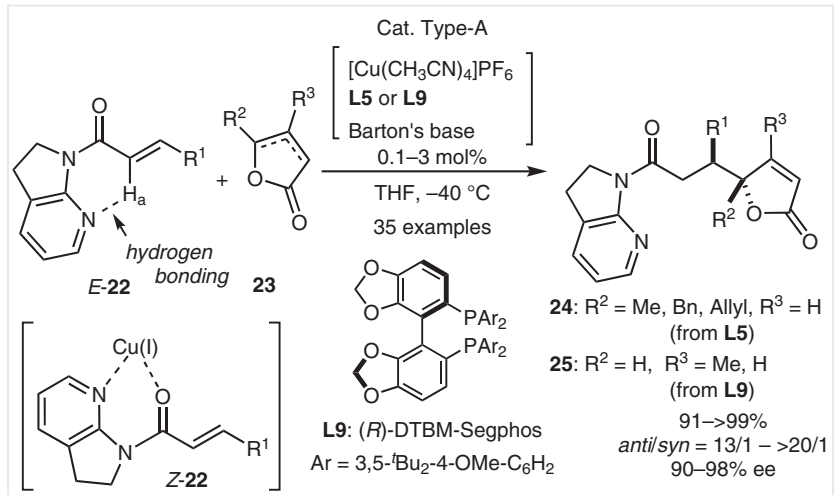

Scheme 19 Catalytic asymmetric conjugate addition of butenolides to $\alpha, \beta$-unsaturated 7-azaindoline amide

\subsection{1,3-Dipolar Cycloaddition Of Nitrones}

Chelation of the 7-azaindoline unit to metal cations elicits enhanced reactivity of the neighboring unsaturated bond as a dipolarophile. In the 1,3-dipolar cycloaddition of nitrones 26, ${ }^{17} \alpha, \beta$-unsaturated 7-azaindoline amide 22 exhibits metal cation accelerated reactivity (Scheme 20). ${ }^{18} \mathrm{Al}-$ though both $\mathrm{Cu}$ (II) and $\mathrm{In}(\mathrm{III})$ complexes promote the reaction, the latter using BHA ligands emerged as more effective promoters.

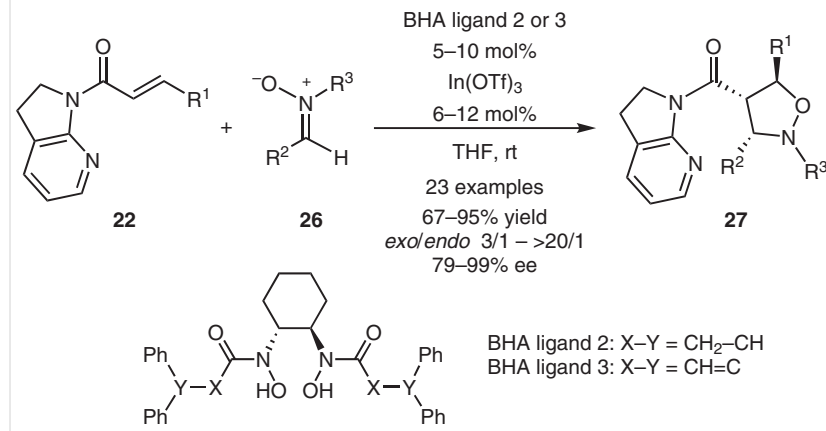

Scheme 20 Catalytic asymmetric 1,3-dipolar cycloaddition of nitrones with $\alpha, \beta$-unsaturated 7 -azaindoline amide

The subtle change in the nitrone structure affects the preferred ligand structure; aromatic nitrones afford the best stereoselectivity with saturated diphenyl-type BHA ligand 2, while unsaturated version BHA ligand 3 outperforms in the reaction with aliphatic nitrones.

\section{Transformation of 7-Azaindoline Amide}

From a synthetic point of view, facile removal of the 7azaindoline attachment is critical (Figure 1). Indeed, divergent transformation is another advantage of the 7-azaindoline amide in the synthesis of versatile chiral building blocks. Reliable transformation into ketones and aldehydes by simple treatment with organometallic reagents and typical hydride reductants is also particularly important because amides sometimes suffer from undesired overalkylation or overreduction. 7-Azaindoline harnesses its chelating ability in stabilizing the tetrahedral intermediate in alkylation and reduction in a similar manner as Weinreb's amide. ${ }^{19}$ Hydrolysis as well as solvolysis are applied via protic or Lewis acidic $(\mathrm{CuCl})$ conditions to give the corresponding acids and esters for further elaboration to meet the synthetic needs.
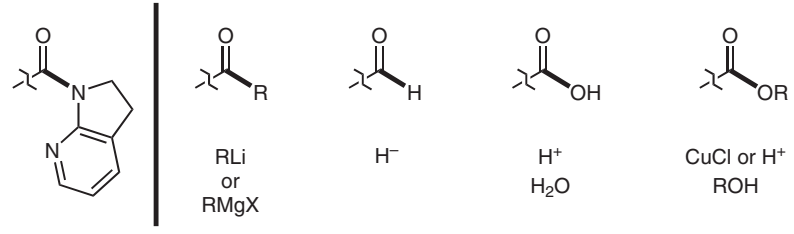

Figure 1 Transformation of the 7-azaindoline amide moiety into various carbonyl-type functional groups

\section{Conclusion}

High-fidelity chelation through the 7-azaindoline amide overrides the effect of other functional groups in substrates, leading to wide substrate generality in nucleophilic and electrophilic catalyses. Inexpensive Cu cations are generally optimal and a number of chiral ligands are broadly available, allowing for the rapid identification of suitable reaction conditions. The easy transformation of the 7-azaindoline amide moiety satisfies the requirements for the attachment; upon efficiently driving the asymmetric catalysis, 7azaindoline is easily cleaved to deliver various carbonyltype chiral building blocks.

\section{Funding Information}

This work was financially supported by ACT-C (JPMJCR12YO) from JST, and KAKENHI (25713002, 17H03025, and JP16H01043 in Precisely Designed Catalysts with Customized Scaffolding) from JSPS. NK thanks The Naito Foundation for financial support.

\section{Acknowledgment}

The authors are grateful to all of the co-workers involved in the research summarized here.

\section{References}

(1) For reviews, see: (a) Alcaide, B.; Almendros, P. Eur. J. Org. Chem. 2002, 1595. (b) Modern Aldol Reactions; Mahrwald, R., Ed.; Wiley-VCH: Weinheim, 2004. (c) Notz, W.; Tanaka, F.; Barbas, C. F. III. Acc. Chem. Res. 2004, 37, 580. (d) Mukherjee, S.; Yang, J. W.; Hoffmann, S.; List, B. Chem. Rev. 2007, 107, 5471. (e) Trost, B. M.; Brindle, C. S. Chem. Soc. Rev. 2010, 39, 1600. (f) Modern Methods 
in Stereoselective Aldol Reactions; Mahrwald, R., Ed.; Wiley-VCH: Weinheim, 2013. (g) Yamashita, Y.; Yasukawa, T.; Yoo, W. J.; Kitanosono, T.; Kobayashi, S. Chem. Soc. Rev. 2018, 47, 4388.

(2) For early seminal works in direct catalytic asymmteric aldol reactions, see: (a) Yamada, Y. M. A.; Yoshikawa, N.; Sasai, H.; Shibasaki, M. Angew. Chem., Int. Ed. Engl. 1997, 36, 1871. (b) Yoshikawa, N.; Yamada, Y. M. A.; Das, J.; Sasai, H.; Shibasaki, M. J. Am. Chem. Soc. 1999, 121, 4168. (c) List, B.; Lerner, R. A.; Barbas, C. F. III. J. Am. Chem. Soc. 2000, 122, 2395. (d) Trost, B. M.; Ito, H. J. Am. Chem. Soc. 2000, 122, 12003.

(3) (a) Arteaga, F. A.; Liu, Z.; Brewitz, L.; Chen, J.; Sun, B.; Kumagai, N.; Shibasaki, M. Org. Lett. 2016, 18, 2391. (b) Liu, Z.; Takeuchi, T.; Pluta, R.; Arteaga, F. A.; Kumagai, N.; Shibasaki, M. Org. Lett. 2017, 19, 710.

(4) (a) Yin, L.; Brewitz, L.; Kumagai, N.; Shibasaki, M. J. Am. Chem. Soc. 2014, 136, 17958. (b) Brewitz, L.; Arteaga, F. A.; Yin, L.; Alagiri, K.; Kumagai, N.; Shibasaki, M. J. Am. Chem. Soc. 2015, 137, 15929. (c) Brewitz, L.; Kumagai, N.; Shibasaki, M. J. Fluorine Chem. 2017, 194, 1. (d) Matsuzawa, A.; Noda, H.; Kumagai, N.; Shibasaki, M. J. Org. Chem. 2017, 82, 8304. (e) Noda, H.; Amemiya, F.; Weidner, K.; Kumagai, N.; Shibasaki, M. Chem. Sci. 2017, 8, 3260. (f) Brewitz, L.; Noda, H.; Kumagai, N.; Shibasaki, M. Eur. J. Org. Chem. 2018, 714.

(5) (a) Sun, Z.; Weidner, K.; Kumagai, N.; Shibasaki, M. Chem.-Eur.J. 2015, 21, 17574. (b) Weidner, K.; Sun, Z.; Kumagai, N.; Shibasaki, M. Angew. Chem. Int. Ed. 2015, 54, 6236.

(6) Sun, B.; Pluta, R.; Kumagai, N.; Shibasaki, M. Org. Lett. 2018, 20, 526.

(7) Sun, B.; Balaji, P. V.; Kumagai, N.; Shibasaki, M. J. Am. Chem. Soc. 2017, 139, 8295.

(8) Weidner, K.; Kumagai, N.; Shibasaki, M. Angew. Chem. Int. Ed. 2014, 53, 6150.

(9) (a) Kanai, M.; Kato, N.; Ichikawa, E.; Shibasaki, M. Synlett 2005, 1491. (b) Yamamoto, H.; Futatsugi, K. Angew. Chem. Int. Ed. 2005, 44, 1924. (c) Paull, D. H.; Abraham, C. J.; Scerba, M. T.; Alden-Danforth, E.; Lectka, T. Acc. Chem. Res. 2008, 41, 655. (d) Acid Catalysis in Modern Organic Synthesis; Yamamoto, H.; Ishihara, K., Ed.; Wiley-VCH: Weinheim, 2008. (e) Kumagai, N.;
Shibasaki, M. Angew. Chem. Int. Ed. 2011, 50, 4760. (f) Cooperative Catalysis; Peters, R., Ed.; Wiley-VCH: Weinheim, 2015.

(10) (a) Tsuda, T.; Yazawa, T.; Watanabe, K.; Fujii, T.; Saegusa, T. J. Org. Chem. 1981, 46, 192. (b) Meyer, E. M.; Gambarotta, S.; Floriani, C.; Chiesi-Villa, A.; Guastinit, C. Organometallics 1989, 8, 1067. (c) Stollenz, M.; Meyer, F. Organometallics 2012, 31, 7708.

(11) (a) Marques, M. M. Angew. Chem. Int. Ed. 2006, 45, 348. (b) Ting, A.; Schaus, S. E. Eur. J. Org. Chem. 2007, 5797. (c) Verkade, J. M.; van Hemert, L. J.; Quaedflieg, P. J.; Rutjes, F. P. Chem. Soc. Rev. 2008, 37, 29. (d) Arrayas, R. G.; Carretero, J. C. Chem. Soc. Rev. 2009, 38, 1940. (e) Kobayashi, S.; Mori, Y.; Fossey, J. S.; Salter, M. M. Chem. Rev. 2011, 111, 2626.

(12) Zhang, W.; Basak, A.; Kosugi, Y.; Hoshino, Y.; Yamamoto, H. Angew. Chem. Int. Ed. 2005, 44, 4389.

(13) (a) Wani, M. C.; Taylor, H. L.; Wall, M. E.; Coggon, P.; McPhail, A. T. J. Am. Chem. Soc. 1971, 93, 2325. (b) Nicolaou, K. C.; Dai, W.M.; Guy, R. K. Angew. Chem., Int. Ed. Engl. 1994, 33, 15. (c) Kingston, D. G. I. Chem. Commun. 2001, 867. (d) Horwitz, S. B. J. Nat. Prod. 2004, 67, 136. (e) Kingston, D. G.; Newman, D. J. Curr. Opin. Drug Discovery Dev. 2007, 10, 130.

(14) (a) Purser, S.; Moore, P. R.; Swallow, S.; Gouverneur, V. Chem. Soc. Rev. 2008, 37, 320. (b) Hunter, L. Beilstein J. Org. Chem. 2010, 6, 38. (c) Zhou, Y.; Wang, J.; Gu, Z;; Wang, S.; Zhu, W.; Acena, J. L.; Soloshonok, V. A.; Izawa, K.; Liu, H. Chem. Rev. 2016, 116, 422. (d) Meanwell, N. A. J. Med. Chem. 2018, 61, 5822.

(15) Zhu, Y.; Han, J.; Wang, J.; Shibata, N.; Sodeoka, M.; Soloshonok, V. A.; Coelho, J. A. S.; Toste, F. D. Chem. Rev. 2018, 118, 3887.

(16) Zhang, M.; Kumagai, N.; Shibasaki, M. Chem.-Eur. J. 2016, 22, 5525.

(17) For reviews, see: (a) Methods and Applications of Cycloaddition Reactions in Organic Syntheses; Nishiwaki, N., Ed.; Wiley: Hoboken, 2014. (b) Klier, L.; Tur, F.; Poulsen, P. H.; Jørgensen, K. A. Chem. Soc. Rev. 2017, 46, 1080.

(18) Zhang, M.; Kumagai, N.; Shibasaki, M. Chem.-Eur. J. 2017, 23, 12450.

(19) For a review, see: Aidhen, I.; Balasubramaniam, S. Synthesis 2008, 3707. 